\title{
Research on Reverse Modeling of Sports Shoes Based on Rhino Software Environment
}

\author{
Peihua Wang \\ Mechanical and electrical technology department, Xijing University Shanxi Xi'an .China \\ andywangpei@126.com
}

Keywords: Reverse engineering; Rhino; Reconstruction; Sports shoes

\begin{abstract}
The intention of this thesis is to explore the concrete realization method of reverse engineering technology in the field of development of sports shoes, how in the rhino software environment, reverse modeling two-demention drawings from product, especially how to generate $3 \mathrm{D}$ reconstruction of product design documents, geometric structure, so as to realize the rapid development of products. Based on the author's many years of work experience, using empirical methods, and examples, combined with a large number of pictures to show sports shoes reverse modeling technology. Through the discussion of the reconstruction process of the geometric structure of the sports shoes, this paper obtains the reverse modeling method and idea of the sports shoes in the rhino environment. In the end we can get the conclusion, if thinking properly, the useing of tools reasonable, reverse modeling sports shoes will be very simple. Through the command commonly used in rhino software environment, especially the flexible use of curves and surface tools, you can easily reconstruct the product geometry.
\end{abstract}

\section{Introduction}

Reverse engineering is the abbreviated form of reverse engineering. Which means a technology that in the absence of design drawings, relying solely on the physical measurement, scanning so as to get data, rebuild the product geometry model, generate the product design files and NC machining program, ang relying on $\mathrm{CAD} / \mathrm{CAM}$ technology to design, manufacture, and thus develop a product. It is different from the traditional designing process which is form drawings to the model to the product, the technology is reversely generated design drawings from the product, so called reverse engineering. After the advent of the technology, science and technology industry of various countries attach great importance to the technology so it gets rapid development[1].

With the development and application of reverse engineering technology, the technology has been applied in many fields, such as architecture, archeology, medical and so on, Its role has also been changed from copying from the original product, into a link of variety of new technologies and one of the important means of rapid product development. The technology and virtual reality, computer aided design, artificial intelligence and other advanced technologies inosculate as a whole, forming the forefront of science and technology[2].

Reverse engineering involves three key technologies or steps, which are as follow:

Data Collection. Data collection is the first step in reverse engineering and is the foundation. The accuracy of data collection to a large extent determine the success or failure of the latter part of the work.

Data Preprocessing. In the process of data collection, due to human factors or various objective conditions, often produce data errors, especially in the edge of the product geometry and some corners, which resulted in the collection of data is not accurate, If this situation is not dealt with, and all the collected data are used, which will inevitably cause the surface is not smooth and the error generated, so for the collection of point cloud data, must be pre-processing. Preprocessing mainly includes format conversion, data filtering and smoothing, data reduction, data segmentation and so on.

Surface Reconstruction. The final purpose of the preliminary work is to reconstruct the geometric surface structure of the product. At present, the common surface reconstruction methods are based on polygon-based surface reconstruction (3DMAX, maya as the representative), mainly 
for the reconstruction of natural organic form. The nurbs-based surface reconstruction (represented by rhino, ug, proe, etc.) is mainly used for the reconstruction of industrial forms.

The reverse modeling of sports shoes, usually divided into two parts of the soles and upper parts. The soles are usually divided into three part which are outsole, insole, footbed. Due to the need for mold of the soles, it often need to collect data by some means, generate two-dimensional drawings, and then reconstruct the geometric structure from the two-dimensional graphics,

\section{The Soles Reverse Modeling Steps are as Follows.}

Import Two-dimensional Projection and Create a Layer. Made two-dimensional graphics according to the data obtained by some device before. The two-dimensional graphics are usually saved as IGS format, and then import the IGS two-dimensional graphics into rhino.

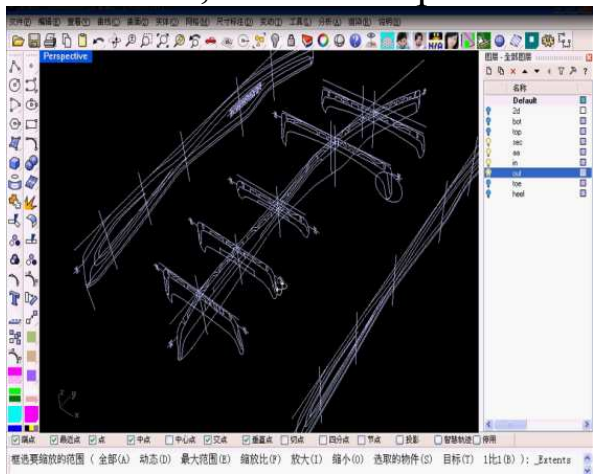

Figure 1.

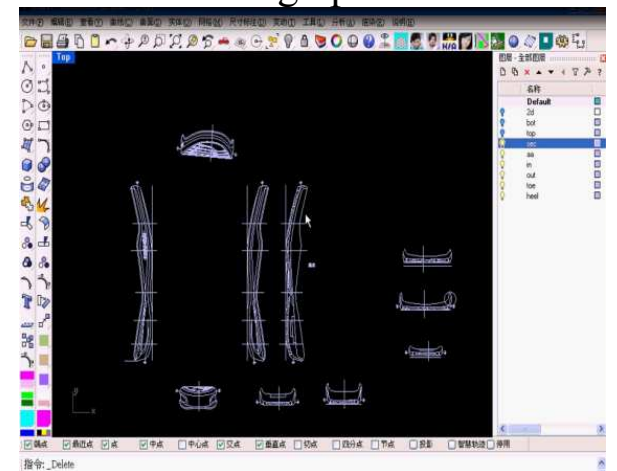

Figure 2.

There are usually front view, back view, bottom view, top view, left view, right view, keel side view, soles cross-section diagram and other two-dimensional projection, rotate the two-dimensional projection and move them to the corresponding projection position, then establishment several layers, put different two-dimensional graphics into corresponding layers.

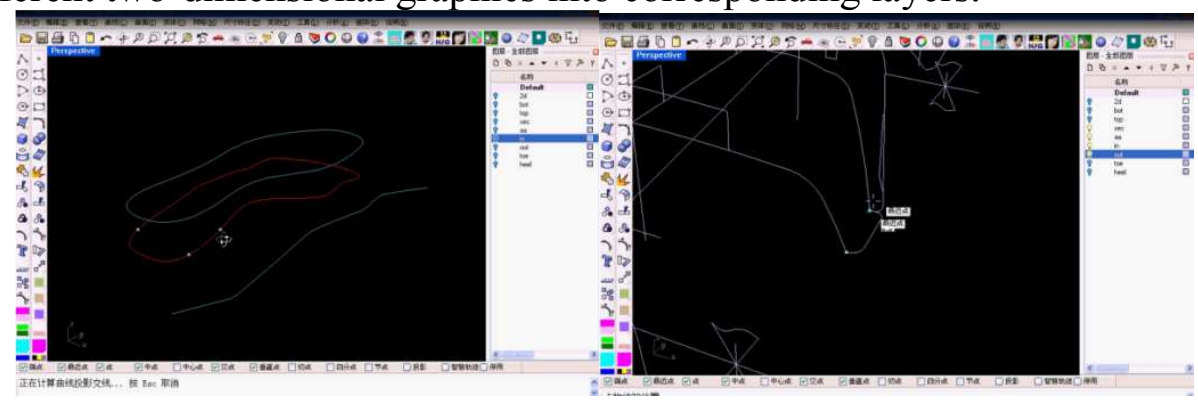

Figure 3.

Figure 4.

Find the Mold Mouth Line of Insole. Draw points in the upper edge of the line of the crosssection of the inshoe, combined with the top view of projection, find the upper edge line of the insoles in the top view of the projection, combined left and right side view of projection,

Find the upper edge line of the insoles in the left view of projection and right view of projection. Uind the insole mold mouth line by the two directions intesect command using the two lines we obtain just now. 


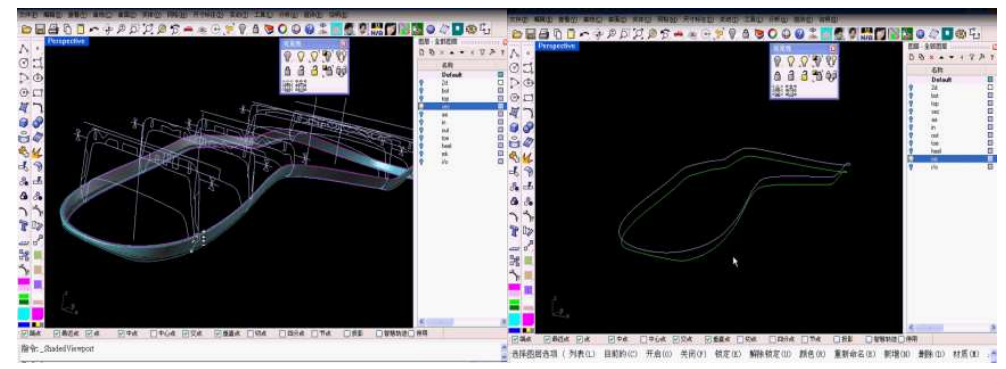

Figure 5.

Figure 6.

Find out the Outsole Mold Mouth Line and Create the side Surface of Insole. The same way to find the upper edge line of the outsole(usually rubber material). And then through the double scan command, combined with the side outlines, scanning the side surface of insole. Measure the fillet radius in a two-dimensional figure and round the face and face with a rounded corner command.

Build the Bottom Surface of Outsoles. Extrude a surface from the outline in the side views of projection of the bottom surface of outsoles, that is the bottom surface of the outsoles, and then in the top view of project to find outsole bottom ourline, This outline usually has been generated in the top two-dimensional projection map after the data collection, then cut off the surface with this outline, .
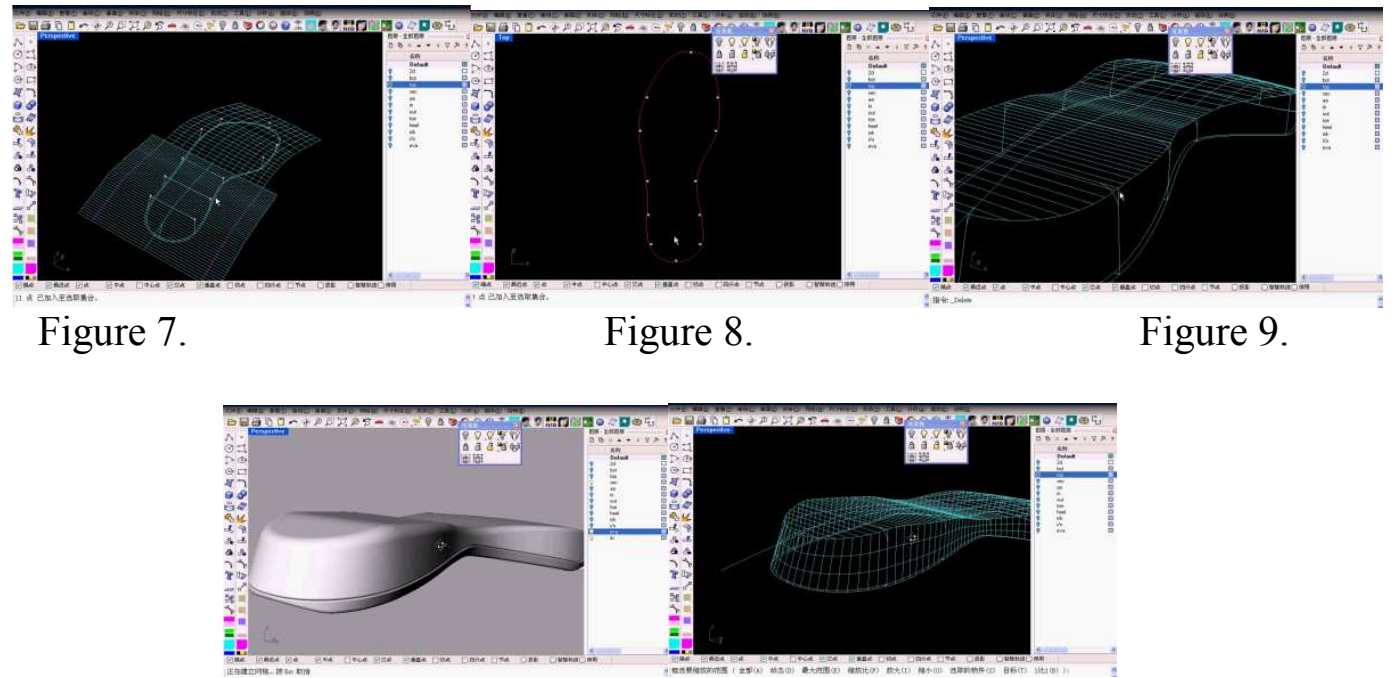

Figure 10.

Figure 11.

Build the Outside Surface of Outsoles. Extracted the bottom surface edge line of outsoles, combine it with the upper surface edge line of outsoles(generated in step3) and side outlines, we can obtain the side surface of outsoles by double scan. Measure the fillet radius between the face and the face in the two-dimensional graph. Fillet between the side surface and the bottom surface of outsoles, then we get all the surface of the outsoles.

Build the Internal Surface of Outsoles. The same way, make the whole internal surface of outsole. At the same time, it also is the outside surface of insole(uaually EVA material), they are fit together. As shown in Fig. 12, so copy a copy.

Make the Bottom Pattern of Outsole. According to the two-dimensional pattern of lines, make the pattern on the outsole. As shown in Fig. 13. 


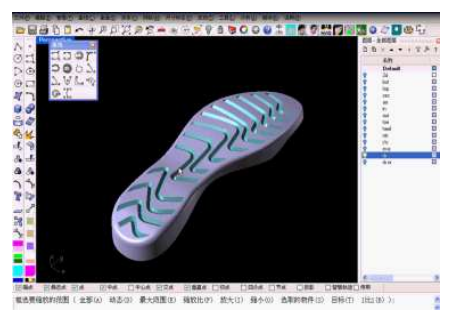

Figure 12.

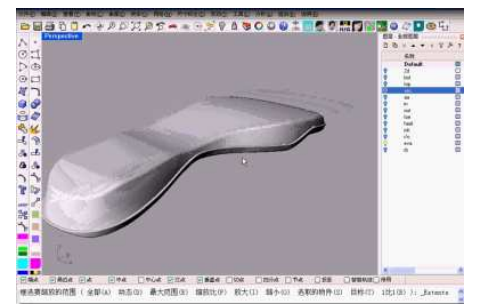

Figure 13.

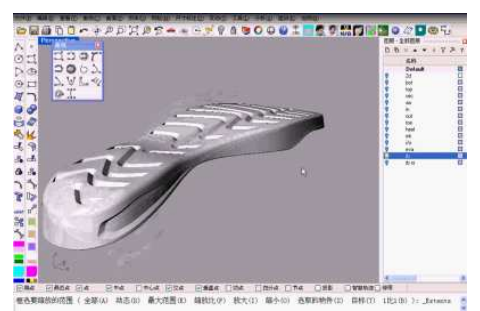

Figure 14.

Make the Side Pattern of outsole. Then, combined with the left and right view of the twodimensional projection map, make the side pattern of outsole. The connection of surface must be smooth, the premise is to line. You can make sure that the connection is smooth by projecting on the surface, drawing lines on the surface, and then mixing. In some cases the outsole is thin, no side pattern, the way to make side pattern is same to above. As shown in Fig. 14.

Unint the Surface of Outsole and Insole into Entity. Up to now, the three sides of the outsole (RB) are ready to be combined into the body, the bottom of insole (EVA) (step 6 copy theinternal surface of the outsole) has been generated, and then using the same method, make the inner surface of the insole, that is, the face in contact with the footbed, and then combined the inner surface of the insole with side surface of insole making in step 3 and the bottom surface of insole making in step 6 into the body. The reverse modeling of the sole is finished.

\section{Reverse Modeling Steps of Upper Shoes.}

The reverse modeling of shoes mainly refers to the modeling of the soles, because the soles need molds, so they need reverse modeling, and the upper generally do not need molding (in addition to a few soles upper is a whole with soles), sticking to the soles after they are made out. So the upper generally use forward modeling. But taking into account the practice, the vamp modeling sometimes is similar to the reverse modeling, but no data collection, just imitating the shape, here is a brief introduction.

Make the Main Surface of Upper Shoes Through Commands Such as Double Scan and Network an So On. Then cut the upper with the line. Draw a line in the top view, cut out the opening of the upper shoe.

Cut out Different Part of the Shoes with the Lines.

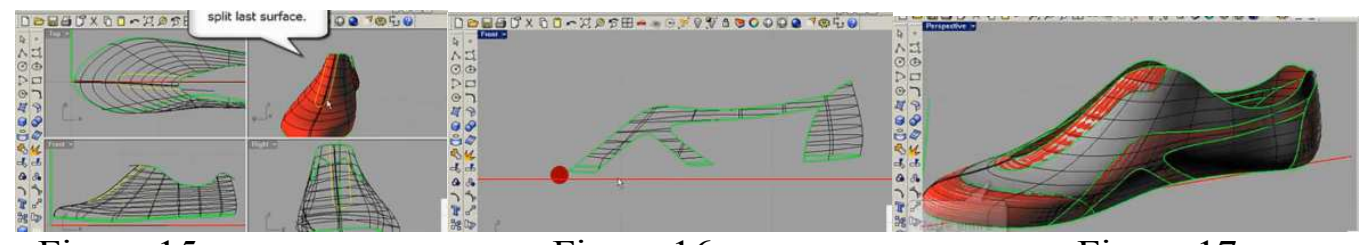

Figure 15.

Figure 16.

Figure 17.

Use the Surface Offset Command to Thicken the Surface and Offset it out of the Shoe. Use double scan or network command to make a connection between surfaces. Transition between surfacees should be smooth. Play shoelaces through the boolean operation, Draw lines, scan to generate shoelaces.

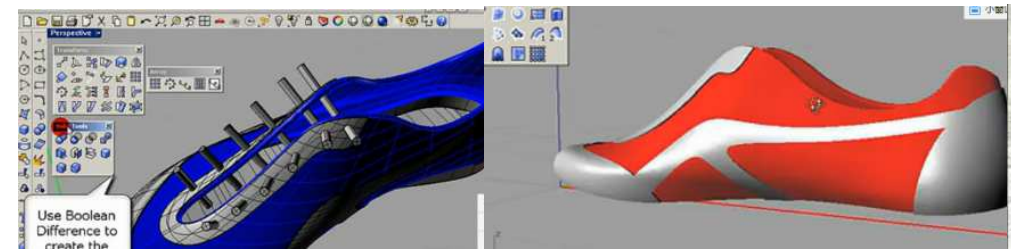

Figure 18.
Figure 19.

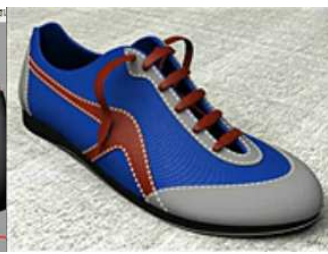

Figure 20. 


\section{Conclusion.}

In summary, in the reverse modeling of sports shoes, reverse modeling technology is widely used in the development of soles due to the current material and process factors. Through data acquisition, data processing, data simplification, data segmentation. Processing the collected data, reconstructiing the geometric structure of the object, so as to achieve reverse modeling, rapid development of new products can be achieve on the basis of this.

\section{Reference}

[1] Tao Jin, Research progress of reverse engineering technology, Beijing, (2002)

[2] Lin Liu, Principle, method and application of reverse engineering, Beijing, (2008),

[3] Lang Su, Rhino 5 complete self-study tutorial, Beijing. first ed. (2013)

[4] Human Education Technology Co Ltd, Ingenious work: Rhino3 product model design, first ed, Tianjin, (2004)

[5] Ding Feng, Top 3D modeling technology, Beijing (2003)

[6] Changyi Yang, Rhino dictionary, Chongqing. first ed. (2015)

[7] Renfei Bai, Rhino 5 digital modeling storm, Beijing. second ed. (2014)

[8] Tiecheng Zhang, Rhino5 product design foundation course, first ed.(2013)

[9] Information on http://www.xuexiniu.com

[10]Information on http://bbs.rhino3d.us 